\title{
Looking for black-holes in X-ray binaries with XMM-Newton: XTE J1817-330 and XTE J1856+053
}

\author{
Gloria Sala, Jochen Greiner and Natalia Primak \\ Max-Planck-Institut für extraterrestrische Physik, PO Box1312, 85741 Garching b.M., Germany
}

\begin{abstract}
The X-ray binary XTE J1817-330 was discovered in outburst on 26 January 2006 with RXTE/ASM. One year later, another X-ray transient discovered in 1996, XTE J1856+053, was detected by RXTE during a new outburst on 28 February 2007. We triggered XMM-Newton target of opportunity observations on these two objects to constrain their parameters and search for a stellar black holes. We summarize the properties of these two X-ray transients and show that the soft X-ray spectra indicate indeed the presence of an accreting stellar black hole in each of the two systems.
\end{abstract}

Keywords: Black holes, X-ray binaries - X-rays: individual: XTE J1817-330, XTE J1856+053

PACS: 97.60.Lf, 97.80.Jp

\section{INTRODUCTION}

X-ray binaries are the brightest X-ray sources in the sky. They are powered by the accretion of material from the secondary star onto a compact object (neutron star or black hole). The accretion regime depends on the spectral type of the companion. In high-mass X-ray binaries (HMXB) the secondary star is an $\mathrm{O}$ or B star with a strong stellar wind which is intercepted and accreted by the compact object. Though an accretion disk may also be present, the secondary star is dominating the optical emission of the source. In low-mass X-ray binaries (LMXB), the secondary star is of a type later than A and the accretion occurs through Loche robe overflow. The optical emission during outburst is in this case dominated by the X-ray heated companion, the outer disk and/or reprocessed hard X-rays.

The generally accepted picture for the X-ray emission of accreting black holes consists of an accretion disk, responsible for thermal black body emission in the X-ray band; and a surrounding hot corona, origin site of non-thermal power-law emission, up to the energy range of gamma-ray telescopes, due to inverse comptonization of soft X-ray photons from the accretion disk.

At present, around $20 \mathrm{X}$-ray binaries contain a dynamically confirmed black hole, and around another 20 are the so called black-hole candidates [1]. Seven of the 20 confirmed black holes, and 12 of the black-hole candidates are transient sources with only one unique outburst observed. 


\section{THE X-RAY TRANSIENTS XTE J1817-330 AND XTE J1856+053}

XTE J1817-330 was discovered by the Rossi X-ray Timing Experiment (RXTE) on 26 January 2006 [2] with a flux of $0.93( \pm 0.03)$ Crab (2-12 keV) and a very soft spectrum, typical for black hole transients. The RXTE light-curve of XTE J1817-330 reached a maximum of 1.9 Crab on 2006 January 28, and then declined exponentially, with an e-folding time of 27 days. Following the initial discovery, the radio, near infrared and optical counterparts were identified [3, 4, 5, 6].

The X-ray transient XTE J1856+053 was discovered with RXTE/PCA during a survey of the Galactic ridge in 1996 [7]. The RXTE/ASM light-curve of the 1996 outburst showed two peaks (Fig. 11): a first symmetric one starting on 1996 April 4, 27 days long in total and with a maximum of $75 \mathrm{mCrab}(2-12 \mathrm{keV})$; and a second fast rise-slow decay (FRED) peak starting on 1996 Sept. 9, lasting for 70 days, and reaching a maximum flux of $79 \mathrm{mCrab}$ [8]. The second X-ray peak was preceded 8 days before by a high-energy (20-100 keV) precursor of 30-60 mCrab detected by BATSE on 1996 Sept. 7-9 [9]. A new outburst of XTE J1856+053 was detected on 2007 February 28 [10]. As in 1996, the RXTE/ASM light curve of the 2007 outburst shows two peaks, preceded by a precursor on 2007 January 10-15 (Fig. 11). The first peak reached a maximum of $\sim 85 \mathrm{mCrab}$ on March 12 and lasted for $\sim 65$ days. The second peak in 2007 rose in only 7 days to $\sim 110$ mCrab, and lasted for $\sim 55$ days. As in the case of the second maximum in 1996, the two 2007 peaks were preceded by hard X-ray precursors detected by Swift/BAT (10-200 keV) in the periods February 22 to March 1, and 2007 May 28-30 [11].

\section{XMM-NEWTON OBSERVATIONS}

Short after the start of the outburst of XTE J1817-330, we triggered a Target of Opportunity Observation (TOO) with XMM-Newton (0.1-10.0 keV) which was executed on the first XMM-Newton visibility window of the source, on 2006 March 13 (obs. ID. 0311590501, $20 \mathrm{ks}$ ), when the source flux detected by the All Sky Monitor (ASM) on board RXTE had faded to $\sim 300 \mathrm{mCrab}[12,13]$.

In a similar way, we obtained a TOO observation of XTE J1856+053 with XMMNewton (0.1-10.0 keV), on 14 March 2007 (obs. ID. 0510010101, 5 ks for RGS, $1.5 \mathrm{ks}$ for EPIC-pn), when the source flux detected by the RXTE/ASM was in its first maximum, $\sim 80 \mathrm{mCrab}$ [14, 15](Fig. 11).

Due to the high brightness of the source, the EPIC-pn camera on board XMM-Newton $(0.2-10.0 \mathrm{keV})$ was used in the burst mode for XTE J1817-330 and in timing mode for XTE J1856+053 to avoid pile-up, while the Reflection Grating Spectrometer (RGS,0.3$2.0 \mathrm{keV}$ ) was operated in spectroscopy high count rate mode. The Optical Monitor (OM) was used in fast mode with the U and UWV1 filters for XTE J1817-330, and with the U filter for XTE J1856+053.

XMM-Newton data were reduced with the XMM-Newton Science Analysis Software v7.1, and XSPEC 11.3 was used for spectral analysis. In the case of XTE J1817-330, the source is clearly detected with the OM both in U and UVW1, with AB magnitudes $\mathrm{U}=15.952 \pm 0.01$ and $\mathrm{UVW} 1=16.13 \pm 0.01$. The EPIC-pn and RGS spectra are fit together in this case with the OM U and UWV1 data, which provide an extra constraint 

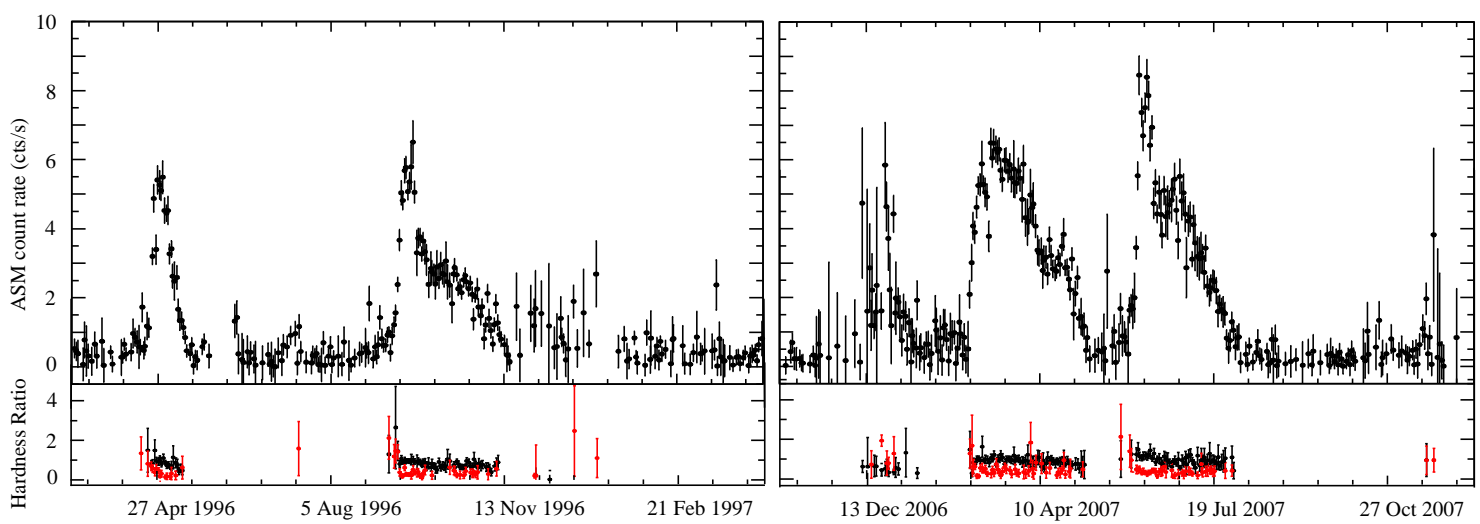

FIGURE 1. RXTE/ASM light-curves and hardness ratios of XTE J1856+053 during its 1996 and 2007 outbursts. The upper panels show the light-curves in the total band $(1.5-12 \mathrm{keV})$. The lower panels show the two hardness ratios: $(3-5 \mathrm{keV}) /(1.5-3 \mathrm{keV})$ is shown in black and $(5-12 \mathrm{keV}) /(3-5 \mathrm{keV})$ is shown in red.

to the absorption and the accretion disk. In the case of XTE J1856+053, the source is not detected in the $\mathrm{OM}$ images, with an upper limit for the $\mathrm{AB}$ magnitude in the $\mathrm{U}$ band during outburst of $23 \mathrm{mag}$.

\section{RESULTS}

We use a 2-component model consisting of a thermal accretion disk plus a comptonization component (compTT model [16]) to fit the spectra of XTE J1817-330 and XTE J1856+053. The standard thermal accretion disk model is composed by the sum of black bodies from surface temperatures depending on the radius as $R^{-3 / 4}$ [17] (diskbb model in $x$ spec). Since this model neglects the torque-free boundary condition, the temperature distribution is not accurate for the innermost disk when it extends down to the innermost stable orbit. The diskpn model in xspec [18] includes corrections for the temperature distribution near the black hole by taking into account the torque-free innerboundary condition. We use here the diskpn model, fixing the inner radius to $6 R_{g}$.

For XTE J1817-330, we obtain the best fit for the diskpn + compTT model $\left(\chi_{v}^{2}=\right.$ 1.18) with $N_{\mathrm{H}}=1.55( \pm 0.05) \times 10^{21} \mathrm{~cm}^{-2}$, a disk with $k T_{\text {in }}=0.70( \pm 0.01) \mathrm{keV}$ and a comptonization component with $\mathrm{kT}_{e}=50 \mathrm{keV}$ (fixed) and $\tau=0.15( \pm 0.02)$. The observed X-ray flux is $8.6( \pm 0.8) \times 10^{-9} \mathrm{erg} \mathrm{cm}^{-2} \mathrm{~s}^{-1}(0.4-10 \mathrm{keV})$, and the unabsorbed $\mathrm{X}$-ray luminosity of the source at the time of the observation $\mathrm{L}_{(0.4-10 \mathrm{keV})}=1.2( \pm 0.1) \times$ $10^{38}(\mathrm{D} / 10 \mathrm{kpc})^{2} \mathrm{erg} \mathrm{s}^{-1}$.

In the case of XTE J1856+053, no indication of a hard component is evident in the residuals, but an excess is present below $1 \mathrm{keV}$, leading to a poor reduced $\chi^{2}$ of 1.99. Adding a recombination emission edge at $0.87 \mathrm{keV}$ (corresponding to O VIII Kshell) with plasma temperature $\mathrm{kT}=50( \pm 3) \mathrm{eV}$ improves the fit. The significance of this feature is however to be taken with care, since the excess could be caused by some redistribution of higher energy photons to lower energies not properly taken into account by the calibration. The best fit $\left(\chi_{v}^{2}=1.16\right)$ is then obtained with $N_{\mathrm{H}}=$ 
$4.5( \pm 0.1) \times 10^{22} \mathrm{~cm}^{-2}$, and a disk (diskpn model) with $k T_{\text {in }}=0.75( \pm 0.01) \mathrm{keV}$. The observed X-ray flux is $1.0( \pm 0.1) \times 10^{-9} \mathrm{erg} \mathrm{cm}^{-2} \mathrm{~s}^{-1}(0.5-10.0 \mathrm{keV})$, which corrected for absorption corresponds to an unabsorbed X-ray luminosity $\mathrm{L}_{(0.5-10.0 \mathrm{keV})}=4.0( \pm 1.5) \times$ $10^{38}(\mathrm{D} / 10 \mathrm{kpc})^{2} \mathrm{erg} \mathrm{s}^{-1}$.

\section{DISCUSSION}

The low temperature of the accretion disk favours a black-hole as the accreting compact object both in XTE J1817-330 and XTE J1856+053. An upper limit for the compact object mass can be obtained from the X-ray spectrum. The normalization constant $K$ of the diskpn model is related to the mass of the compact object $M$, the distance to the source $D$, and the inclination $i$ of the disk as $K=\frac{M^{2} \cos (i)}{D^{2} \beta^{4}}$, where $\beta$ is the color/effective temperature ratio. Furthermore, the accretion rate can be obtained from the mass of the compact object and the maximum temperature of the disk [18].

Assuming $\beta=1.7$ and using the best fit value for the normalization of the diskpn model for XTE J1817-330 $\left(K_{\text {diskpn }}=0.024 \pm 0.002\right)$, we can compare the accretion rate for different possible masses, distances, and inclinations with an upper limit for the accretion rate. At the time of the XMM-Newton observation of XTE J1817-330, the flux of the source had decreased by a factor 6 with respect to the maximum registered by RXTE. Taking the Eddington limit as the upper limit for the accretion rate at the maximum of the outburst, the accretion rate at the time of the observation could not be higher than $16 \%$ of $\mathrm{M}_{\mathrm{Edd}}^{\mathrm{acc}}$. This sets an upper limit for the mass of the central object of $6 \mathrm{M}_{\odot}$ (see [13] for more details).

In the case of XTE J1856 $+053, K_{\text {diskpn }}=(8.5 \pm 0.4) \times 10^{-3}$. At the time of the XMMNewton observations, the flux of the source was at the maximum of the first outburst detected in March 2007. However, a brighter outburst was detected by RXTE/ASM in June 2007. At the time of our XMM-Newton observations, the flux was $70 \%$ of the maximum detected in June 2007. Taking the Eddington limit as the upper limit for the accretion rate at the maximum in June 2007, the accretion rate at the time of the XMMNewton observation could not be higher than $0.7 \mathrm{M}_{\text {Edd }}^{\text {acc }}$. This sets an upper limit for the mass of the central object of $4.2 \mathrm{M}_{\odot}$.

We note, however, that our estimation for the upper limits of the compact object mass depends on the color correction factor. The value adopted here $(\beta=1.7)$ is rather conservative in comparison, for example, to $\beta=2.6$ obtained by Shrader \& Titarchuk [19]. A larger color correction factor would, of course, imply a larger upper limit for the black hole mass.

The absorption column obtained for XTE J1817-330 is in agreement with the low absorption already pointed out at the discovery of the source with RXTE [2]), and confirmed later in UV and X-rays [20, 21, 22, 23]. The low absorption towards XTE J1817330 makes this new black hole candidate an ideal target for optical observations in the quiescent state, which could provide the dynamical confirmation of the presence of a black hole in the system.

The type of the secondary star can be constrained from the non-detection of the sources in quiescence. XTE J1817-330 was not detected previous to the outburst in the 

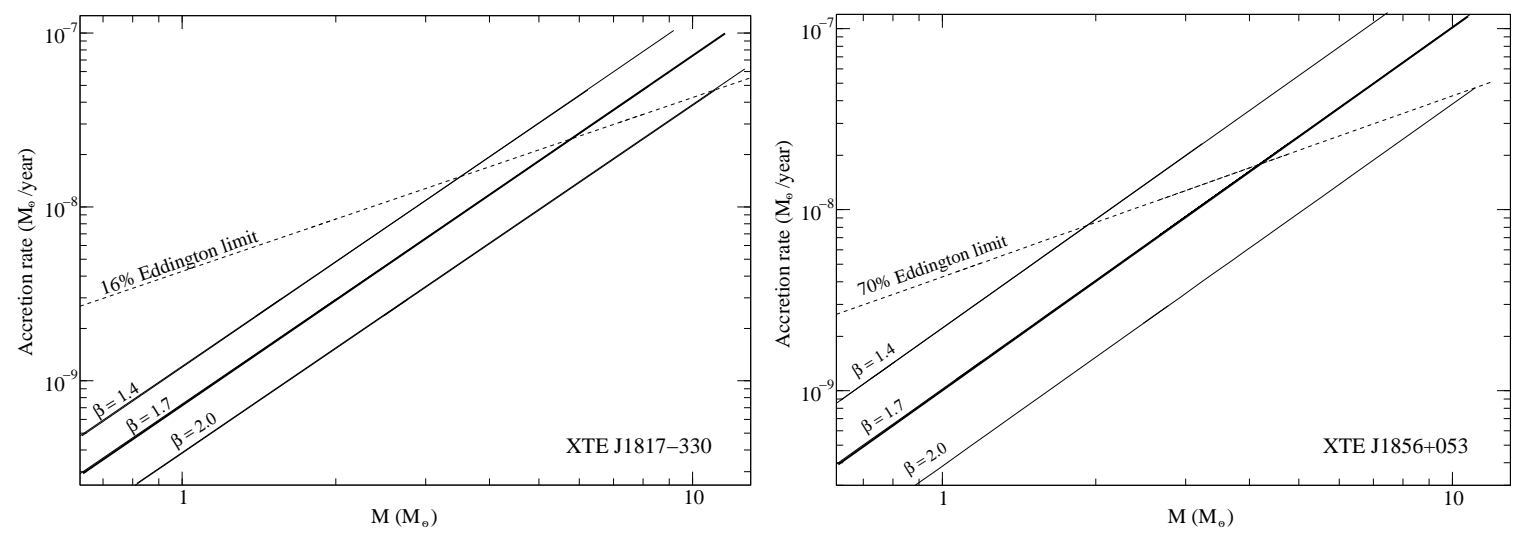

FIGURE 2. Accretion rate as a function of the black hole mass derived from the X-ray spectral fit of XTE J1817-330 (left) and XTE J1856+053 (right). Over plotted is shown the accretion rate upper limit obtained by assuming that at maximum the source was at the Eddington limit and scaling it down to the $\mathrm{X}$-ray flux at the time of the XMM-Newton observation.

digitized sky survey (DSS, $\mathrm{V}>22 \mathrm{mag}$ ). With the extinction towards the source derived from the $\mathrm{N}_{\mathrm{H}}, \mathrm{A}_{\mathrm{V}}=0.76$ (using $N_{\mathrm{H}}=5.9 \times 10^{21} E_{B-V} \mathrm{~cm}^{-2}$ [25]), the lower limit on the absolute magnitude is $\mathrm{M}_{\mathrm{V}}>6 \mathrm{mag}$ (for $10 \mathrm{kpc}$ ). A limit on the absolute magnitude of $\mathrm{M}_{\mathrm{V}}>6$ mag implies that the secondary star must be a K-M star, and a giant can be excluded, even for a $10 \mathrm{kpc}$ distance. We therefore conclude that XTE J1817-330 is a low-mass X-ray binary.

XTE J1856+053 was observed on 26 August 2007 with GROND [24], a 7-channel imager mounted at the MPI/ESO 2.2m telescope at La Silla (Chile). The source was not detected in a 8-minute exposure, with lower limits for the IR magnitudes of $\mathrm{J} \sim 20$, $\mathrm{H} \sim 19$, and $\mathrm{K} \sim 18$ (calibrated against 2MASS). This rules out the presence of a massive secondary in XTE J1856+053. Even for a distance of $10 \mathrm{kpc}$, an O or B star would be detected with $\mathrm{J} \sim 9-11$. We can thus classify XTE $\mathrm{J} 1856+053$ as a low mass X-ray binary (LMXB). We can also use the GROND non-detection to estimate a lower limit for the distance: for a faint M2 secondary (the latest spectral type of a donor in a confirmed $\mathrm{BH}$ binary; see Tab. 1 in McClintock \& Remillard [26]), and with the extinction derived from the X-ray absorption column $\left(\mathrm{A}_{J}=6.7\right.$, using $N_{\mathrm{H}}=5.9 \times 10^{21} E_{B-V} \mathrm{~cm}^{-2}$ and $\left.\mathrm{A}_{J}=0.282 \mathrm{~A}_{V}[25,27]\right)$ the non-detection in the $\mathrm{J}$ band would set a lower limit for the distance of $1 \mathrm{kpc}$.

\section{ACKNOWLEDGMENTS}

We thank Norbert Schartel and the XMM-Newton team for carrying out the TOO observations presented here. We acknowledge the RXTE/ASM team for the public availability of the quick-look results. XMM-Newton is an ESA Science Mission directly funded by ESA Member States and the USA (NASA), with support from BMWI/DLR (FKZ 50 OX 0001) and the Max-Planck Society. GS is supported through DLR (FKZ 50 OR 0405). 


\section{REFERENCES}

1. Remillard \& McClintock, ARA\&A 44, 49 (2006)

2. Remillard et al. ATel\#714 (2006)

3. Rupen, Dhawan, \& Mioduszewski, ATel\#717 (2006)

4. Rupen, Dhawan, \& Mioduszewski, ATel\#721 (2006)

5. D'Avanzo et al., ATel\#724 (2006)

6. Torres et al. ATel\#733 (2006)

7. Marshall et al. IAUC\#6504 (1996)

8. Remillard, Mem.S.A.It 70, 881 (1999)

9. Barret et al. IAUC\#6519 (1996)

10. Levine et al. ATel\#1024 (2007)

11. Krimm et al. ATel \#1093 (2007)

12. Sala \& Greiner, ATel\#791 (2006)

13. Sala, Greiner, Ajello, Bottacini, \& Haberl, $A \& A$, 473, 561 (2007)

14. Sala, Greiner, \& Haberl, ATel\#1062 (2007)

15. Sala, Greiner, Ajello \& Primak, $A \& A$, submitted

16. Titarchuk, ApJ 434, 313 (1994)

17. Mitsuda et al.PASJ 36, 741 (1984)

18. Gierliński et al. MNRAS 309, 496 (1999)

19. Shrader \& Titarchuk, ApJ, 598, 168 (2003)

20. Miller et al. ATel\#743 (2006)

21. Steeghs et al. ATel\#740 (2006)

22. Miller et al. ATel\#746 (2006)

23. Goldoni et al. ATel \#742 (2006)

24. Greiner et al. PASP 120, 405 (2008)

25. Zombeck, Handbook of Space Astronomy and Astrophysics (Cambridge University Press, 3rd ed.) (2007)

26. McClintock \& Remillard, in "Compact Stellar X-ray Sources", eds. W.H.G. Lewin \& M. van der Klis, CUP, chap. 4 (2004)

27. Rieke \& Liebofsky,ApJ 288, 618 (1985) 